\title{
Exploring Helium Mitigation in Ferritic Alloys by Advanced Microscopy
}

Chad M. Parish ${ }^{1}$, Philip D. Edmondson ${ }^{1}$, Fred W. Meyer ${ }^{2}$, Mark E. Bannister ${ }^{2}$, Baishakhi Mazumder ${ }^{3}$, and Michael K. Miller ${ }^{3}$

1. Materials Science and Technology Division, Oak Ridge National Laboratory, Oak Ridge, TN USA

2. Physics Division, Oak Ridge National Laboratory, Oak Ridge, TN USA

3. Center for Nanophase Materials Sciences, Oak Ridge National Laboratory, Oak Ridge, TN USA

Future nuclear energy systems, fission or fusion, will show significantly higher-energy ("harder") neutron spectra than the thermal-spectrum reactors of today. Hard neutron spectra make the heliumgenerating $(n, \alpha)$ reaction more pronounced. Coupled with high operating temperatures, helium embrittlement may become the lifetime-limiting failure mode [1]. Materials design paradigms to provide helium mitigation need to be explored experimentally by high-resolution microscopy. Here, we discuss STEM and APT-based methods to relate microstructural features such as nanoclusters (NCs) and carbides to nanometer-scale helium bubbles.

Samples were F82H or 14YWT ferritic alloys. F82H contains multiple carbide populations and 14YWT multiple oxide populations, including 2 nm-scale NCs [2,3]. Samples were irradiated with a stackedenergy He beam to $\sim 8000$ ppm and «1 dpa [4] or monochromatic He to 30,000 appm at $~ 1$ dpa [3] at multiple temperatures. From APT isodensity surfaces, we previously demonstrated imaging of He bubbles, and employed it to measure if they were associated with NCs [3]. We have also recently found accumulation of He bubbles to $\mathrm{M}_{23} \mathrm{C}_{6}$-type carbides in F82H [4], Fig. 1.

TEM is traditionally used to image voids of bubbles via over/under focused conditions. However, resolution is limited to $\sim 2 \mathrm{~nm}$ [5]. HAADF in STEM provides higher resolution, but it is difficult to differentiate bubbles from NCs in these alloys in HAADF mode. Combining BF-STEM in over- or under-focus [6] with in-focus HAADF makes clear differentiation of bubbles / voids from NCs possible, Fig. 2. Further, recent advances in EDS X-ray mapping capability [7] make it possible to map chemically the extremely small NCs or carbides, Fig. 3, allowing the highest level of differentiation between the different features in the materials' helium-damaged microstructure.

In summary, determining the size, density, and distribution of He bubbles is necessary for successful deployment of future nuclear power systems. Combining advanced STEM and APT methods allows comprehensive characterization of the He bubble distributions in irradiated materials. [8]

[1] SJ Zinkle \& NM Ghoniem, Fus. Eng. Des., V51 (2000) P.55.

[2] RL Klueh et al., Curr. Op. Sol. State Mater. Sci., V8 (2004) P.239.

[3] PD Edmondson et al., J. Nucl. Mater., V434 (2013) P. 210.

[4] B Mazumder et al., Nucl. Mater. and Energy, 1 (2015) in press.

[5] B. Yao et al., J. Electron Micros., V61 (2012) P. 393.

[6] CM Parish \& MK Miller, Microsc. Microan., V20 (2014) P. 568.

[7] P Schlossmacher et al., Micros. Today, July (2010) P. 14.

[8] This work was supported by the U.S. Department of Energy, Office of Science, Basic Energy Sciences, Materials Sciences and Engineering Division. A portion of the Microscopy was conducted as part of a user proposal at ORNL's Center for Nanophase Materials Sciences, which is an Office of Science User Facility. We acknowledge the use of the Analytical Instrumentation Facility (AIF) at North 
Carolina State University, which is supported by the State of North Carolina and the National Science Foundation. We thank Dr. D. T. Hoelzer for 14YWT sample material.

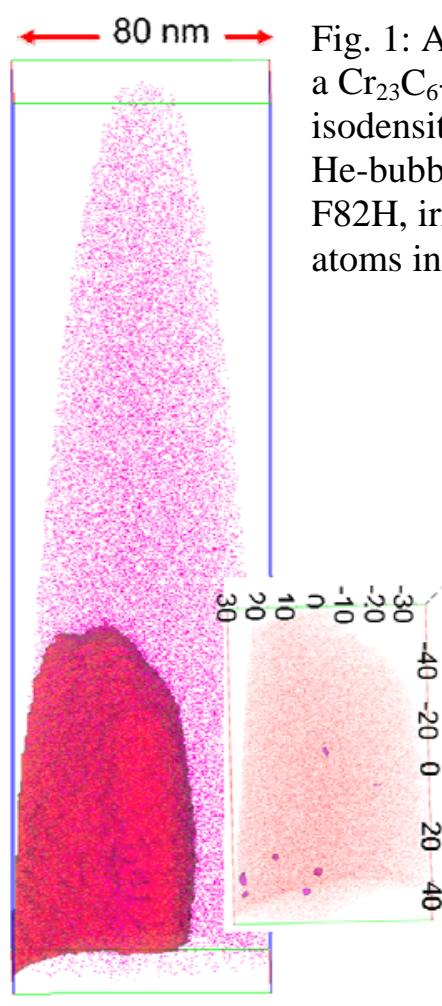

Fig. 2: Aberration-corrected STEM of He-bubbles. Left: underfocus BF with small collection angle $\left(\beta_{\mathrm{BF}}\right)$; right, HAADF image. Bubbles can be matched between the two modes. ORNL Titan microscope. $82 \mathrm{H}$, irradiated at $\sim 50-60^{\circ} \mathrm{C}$. $\mathrm{Cr}$ toms in purple.

\section{$\Delta f_{N}+100 \mathrm{~nm} \quad \beta_{\mathrm{BF}}=1.1 \mathrm{mrad}$}
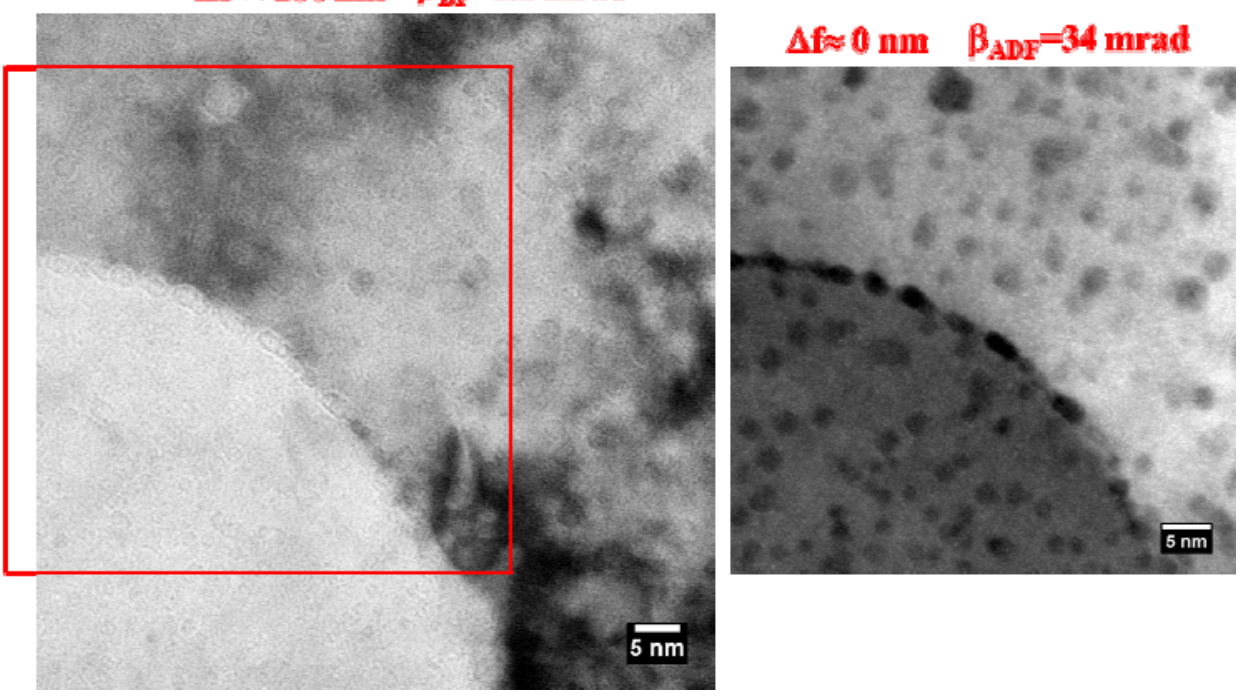

Fig. 3: High-resolution aberration-corrected HAADF (left) and X-ray map (right) of He-irradiated 14YWT. (This region is between the ion-incidence surface and He stopping band, so no bubbles are seen.) X-ray map colored by multivariate statistical analysis (MVSA): Red: Fe-Cr-W matrix; Green: Cr-W grain boundary segregation; Blue: Y-Ti-O rich nanoclusters. North Carolina State University Titan Microscope.
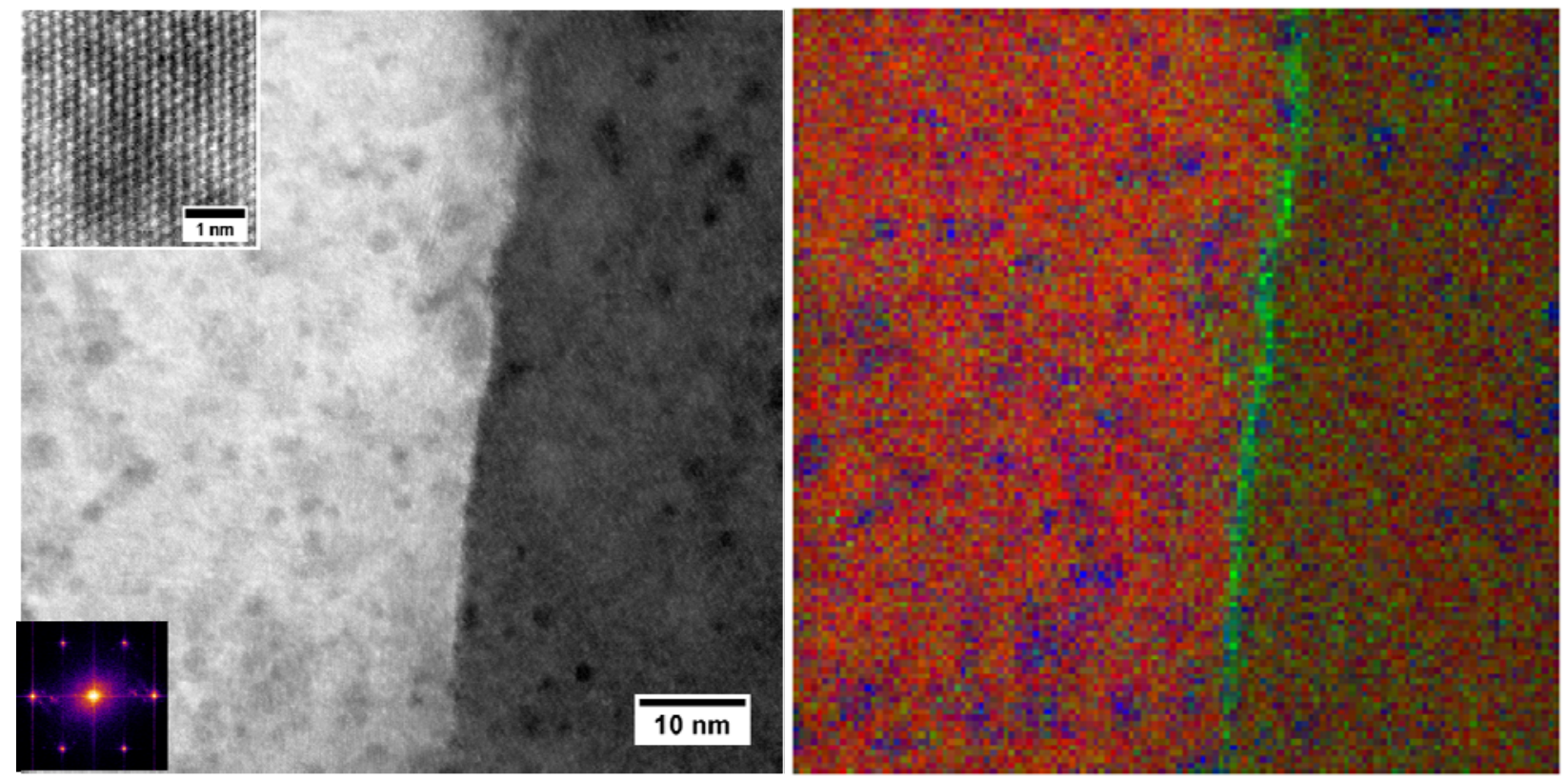